\title{
In Search of the Mysterious Alzheimer's Disease
}

\section{Gabriela Beatriz Acosta*}

Institute of Pharmacological Research (ININFA), National Scientific and Technologic Research Council (CONICET) and University of Buenos Aires (UBA), Argentina

\begin{abstract}
This article present the complexity of Alzheimer's Disease (AD) improves, the biological bases underlying its pathogenesis are gradually being disclosed, and we can expect that new therapeutic targets will emerge. AD is characterized behaviorally by progressive memory loss and cognitive decline and physiologically by the presence of beta-amyloid peptide (A $\beta$ ) and Neurofibrillary Tangles (NFT) in the brain. The aim is to prevent or at least slow down the progression towards clinical impairment. The pathological mechanisms implicated the actions of $\beta$-amyloid, the accumulation of aggregates, the inflammatory cascade, oxidative neuronal damage, tau protein alterations and the formation of NFT, synaptic failure and neurotransmitter depletion. Several of these events are common to many slowly progressive neurodegenerative disorders. The familial forms of Alzheimer's, secondary to inherited mutations have provided an insight into the molecular mechanisms implicated in disease pathogenesis.
\end{abstract}

The underlying cause of $A D$, as well as its treatment, is still under investigation. A number of valuable diagnostic tools have been developed and continue to be improved. Risk factors for AD include age, genetic predisposition, environmental factors, cardiovascular diseases, diabetes and diet.

Keywords: Amyloid precursor protein; Cell death; Neurodegeneration; Oxidative damage; Memory loss; Genetic predisposition; Neurofibrillary tangle; Neuritic plaque; Treatments; Social impact

Abbreviations: FDG: 2- $\left[{ }^{18} \mathrm{~F}\right]$ fluoro-2-deoxy-D-glucose; AD: Alzheimer's Disease; AICD: Amiloid Intracelullar Domain; APP: Amyloid Precursor Protein; ASL: Amyotrophic Lateral Sclerosis; ApoE: Apolipoprotein E; CSN: Central Nervous System; MRI: Magnetic Resonance Imaging; MTL: Medial Temporal Lobes; NFT: Neurofibrillary Tangles; PET: Positron Emission Tomography; BACE1: Protein-cleaving enzyme $1 ; A \beta: \beta$-amyloid Peptide

\section{Introduction}

Alzheimer's Disease (AD) was first described by German psychiatrist and neuropathologist, Alois Alzheimer in 1906 and was named after him [1-3]. AD is the most common form of dementia in the elderly human and one of the most serious health problems in the industrialized world. The elderly are the most rapidly growing part of the population, and increases in life expectancy will inevitably lead to a further increase in the prevalence of $\mathrm{AD}$. At this moment, $\mathrm{AD}$ afflicts $10 \%$ of individuals over 65 years of age, and more than $50 \%$ of persons over 80 years, with age-associated cognitive impairments affecting 10 times as many individuals [4].

Although AD develops differently for every individual, there are many common symptoms [5]. Early symptoms are often mistakenly thought to be "age-related" involves or indicator of stress [6]. In the early stages, the most common symptom is difficulty in "remembering recent events". When AD is suspected, the diagnosis is usually confirmed with assessment that evaluate behavior and thinking abilities, often followed by a brain scan if available [7]. There is no cure for this disease, which worsens as it progresses, and eventually leads to death.

\section{What are the Differences between Degenerative and Neurodegenerative Diseases?}

Neurodegenerative diseases are heterogeneous in their pathological symptoms or anatomical findings (e.g., the differences between AD and Amyotrophic Lateral Sclerosis (ASL)). Do not know the causes of the loss of neurons in neurodegenerative diseases. In other pathologies, such as demyelization in ALS or metabolic disorders are called degenerative diseases, because the loss of neurons are known to cause.
In general, patients with neurodegenerative diseases know when symptoms starts, but not when the sicknesses begin, such as when neurons initiated to die. Many functional systems in CNS are redundant and they all have more or less functional reserve. Thus, symptoms initiate when the remaining neurons are unable to compensate those who have disappeared.

Several studies have shown that the progression of AD pathology in the brain can be staged and might be alters associated with the cognitive decline characteristic of AD develop. Many years before the clinical manifestations of the disease become apparent using standard approaches to assessment [8-11].

The adequate classification of diseases in Medicine is essential for a correct diagnosis and prognosis of patients. Currently, the most common cataloging of neurodegenerative diseases it based on the topography of the lesion and/or clinical appearance. There are key areas of CNS where neurons are lost or degenerate, usually in cerebral cortex, basal ganglia, brain stem, cerebellum and spinal cord.

With the development of specific and effective prevention treatments of $\mathrm{AD}$ will be increasingly critical for medical care. As strategies to delay disease progression, and possibly prevent or offset the onset of $\mathrm{AD}$ are under development [12], it is extremely important to recognize individuals at high risk for developing $\mathrm{AD}$ who may particularly benefit from early therapeutic interventions.

The definitive diagnosis of $\mathrm{AD}$ is based on the post-mortem observation of specific pathological intracellular lesions, neurofibrillary tangles (NFT), $\beta$-amyloid deposition in the form of extracellular senile plaques and blood vessel deposits, synapse dysfunction $[8,9,13,14]$. AD

*Corresponding author: Gabriela Beatriz Acosta, Institute of Pharmacological Research (ININFA), National Scientific and Technologic Research Council (CONICET) and University of Buenos Aires (UBA), Junín 956, 5th floor, C1113AAD Buenos Aires, Argentina, E-mail: gcosta@ffyb.uba.ar

Received November 23, 2012; Accepted December 28, 2012; Published December 31, 2012

Citation: Acosta GB (2013) In Search of the Mysterious Alzheimer's Disease. Clin Exp Pharmacol S6:001. doi:10.4172/2161-1459.S6-001

Copyright: (c) 2013 Acosta GB. This is an open-access article distributed under the terms of the Creative Commons Attribution License, which permits unrestricted use, distribution, and reproduction in any medium, provided the original author and source are credited. 
pathology translates to functional and structural brain damage, mainly involving the brain regions where the concentration of cell loss is the greatest in $\mathrm{AD}$ [15].

The major circuits in AD disease are structurally disrupted through synaptic loss and neuronal death. There are a selective vulnerability between neurons die and neurons resistant to neurodegeneration [15]. NFT deposition originates in the Medial Temporal Lobes (MTL: hippocampus, transentorhinal, entorhinal cortex, parahippocampal gyrus), with a role in the neural control of memory functions. Then, begin to cluster in the adjacent inferior temporal and posterior cingulate cortex in mild $\mathrm{AD}$, and finally spread to the parieto-temporal and prefrontal association cortex, which are involved in the neural control of perception, attention and language, in mild and subsequent severe dementia $[8,11]$

Neuroimaging techniques offer the opportunity to track AD-related brain changes in vivo, which is critical for creating an early diagnosis capacity. Substantial neuronal loss results in structural brain modifies that can be visualized as volume reductions (i.e., atrophy) by using Magnetic Resonance Imaging (MRI), and in functional abnormalities, especially as alterations in the metabolic rate of glucose consumption, which can be measured with Positron Emission Tomography (PET) imaging using $\left.2-{ }^{18} \mathrm{~F}\right]$ fluoro-2-deoxy-D-glucose (FDG) as the tracer. FDG-PET has the unique ability to provide quantitative estimates the local cerebral metabolic rate of glucose [16,17] (Figure1 \& Figure 2).

\section{Proteins Abnormalities in Alzheimer's Disease}

\section{$\beta$-Amyloid}

Cerebral plaques loaded with $\beta$-amyloid peptide $(A \beta)$ and dystrophic neuritis in neocortical terminal fields as well as prominent NFT in MTL structures are important pathological features of AD. Loss of neurons and white matter, inflammation and oxidative damage are also present. $A \beta$ peptides are natural products of metabolism consisting of 36 to 43 amino acids. Monomers of $A \beta 40$ are much more

\section{POST-MORTEM STAINED BRAIN SECTIONS}
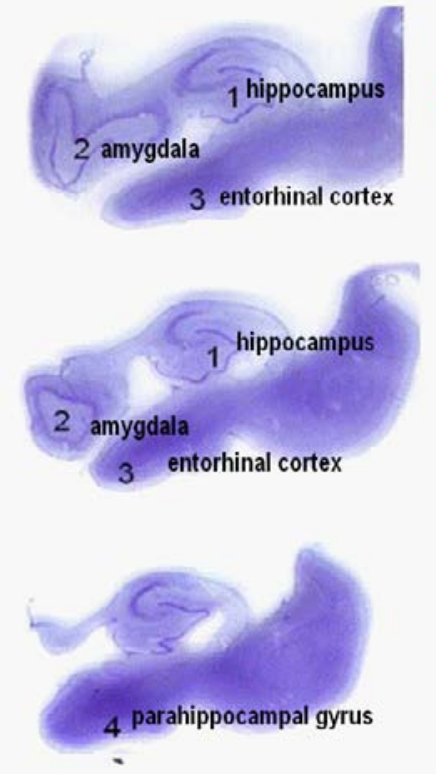

Figure 1: Neurofibrillary deposition originates in the medial temporal lobes.

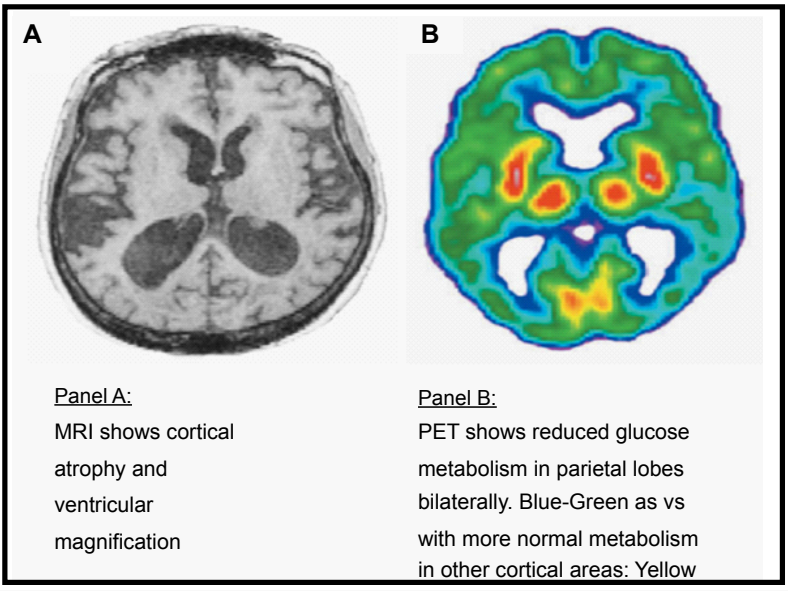

Figure 2: Scans of two patients, with possible Alzheimer's Disease.

prevalent than the aggregation-prone and damaging $A \beta 42$ species. $\beta$-amyloid peptides originate from proteolysis of the amyloid precursor protein by the sequential enzymatic actions of $\beta$-site amyloid precursor protein-cleaving enzyme 1 (BACE-1), a $\beta$-secretase, and $\gamma$-secretase, a protein complex with presenilin 1 at its catalytic corea. An imbalance between production and clearance, and aggregation of peptides, causes $\mathrm{A} \beta$ to accumulate, and this excess may be the initiating factor in $A D$, including Down's syndrome, and evidence that $A \beta 42$ is toxic to cells. $\mathrm{A} \beta$ spontaneously self-aggregates into multiple coexisting physical forms [18]. One form consists of oligomers (2 to 6 peptides), which come together into intermediate assemblies $\beta$-amyloid can also grow into fibrils, which arrange themselves into $\beta$-pleated sheets to form the insoluble fibers of advanced amyloid plaques. Soluble oligomers and intermediate amyloids are the most neurotoxic forms of levels of synaptic $\mathrm{A} \beta$ may dampen excitatory transmission and prevent neuronal hyperactivity [18].

The amyloid hypothesis: The central event for the amyloid cascade hypothesis is the excessive formation of $A \beta$. The source of excessive $\mathrm{A} \beta$ formation is the amyloid precursor APP and multiple factors may contribute to its aberrant processing. Here we have indicated genetic alterations since it has been largely demonstrated that mutations associated with familial forms of $\mathrm{AD}$, result in aberrant metabolism and excess production of $A \beta$. Defective signal transduction mechanisms, neurotransmitter changes and other perturbations of normal cellular homeostasis may also contribute to aberrant APP processing. Most of these alterations have been detected and characterized in the brain and/ or peripheral tissues of $\mathrm{AD}$ patients and constitute an example of the regulated processing of APP pharmacological [18] (Figure 3).

\section{Tau}

NFT, which are filamentous inclusions in pyramidal neurons, occur in $\mathrm{AD}$ and other neurodegenerative disorders termed tauopathies [19]. The number of NFT is a pathologic marker of the severity of AD. The major component of the tangles is an abnormally hyperphosphorylated and aggregated form of tau. Normally an abundant soluble protein in axons, tau promotes assembly and firmness of microtubules and vesicle transport. Hyperphosphorylated tau is insoluble, lacks affinity for microtubules, and self-associates into paired helical filament structures. Enzymes that add and those that remove phosphate residues regulate the level of tau phosphorylation [20]. Like $A \beta$ oligomers, intermediate aggregates of abnormal tau molecules are cytotoxic and impair 


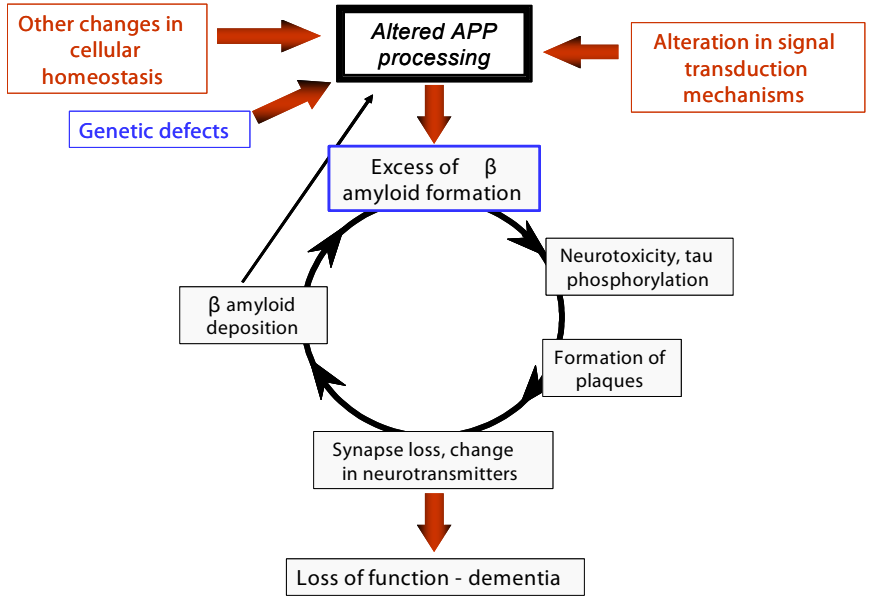

Figure 3: APP processing regulation.

cognition. Insoluble helical filaments may be inert, however, since decreases in axonal transport and neuron number are independent

There is evidence that the volume loss detected on MRI is related to both the extent of NFT pathology and to the magnitude of neuronal loss [21,22]. AD pathology is known to have the general effect of disrupting axonal transport and inducing metabolic declines. $A \beta$ is a fragment from a larger protein called Amyloid Precursor Protein (APP), a transmembrane protein that penetrates through the neuron's membrane. APP is critical to neuron growth, survival and post-injury repair $[23,24]$. In $A D$, an unknown process causes APP to be divided into smaller fragments by enzymes through proteolysis. Also APOE4, the major genetic risk factor for $\mathrm{AD}$, leads to excess amyloid buildup in the brain [25] (Figure 4).

\section{The Synapse in Alzheimer's Disease}

AD may be a primarily a disorders of synaptic failure. The oldest, on which most currently available drug therapies are based, is the cholinergic hypothesis which proposes that $\mathrm{AD}$ is caused by reduced synthesis of the Neurotransmitter (NT) acetylcholine. The cholinergic hypothesis has not maintained widespread support, largely because medications intended to treat acetylcholine deficiency have not been very effective. Other cholinergic effects have also been proposed, for example, initiation of large-scale aggregation of amyloid leading to generalized neuroinflammation [26].

Reduction in the activity of the cholinergic neurons is a well-known feature of Alzheimer's disease [27] Acetylcholinesterase inhibitors are employed to reduce the rate at which acetylcholine $(\mathrm{ACh})$ is broken down, thereby increasing the concentration of $\mathrm{ACh}$ in the brain and combating the loss of ACh caused by the death of cholinergic neurons. Cholinesterase inhibitors approved for the management of AD symptoms are donepezil (Aricept), galantamine (Razadyne) [28] and rivastigmine (Exelon). The most common side effects are nausea and vomiting, both of which are linked to cholinergic excess. These side effects arise in approximately $10-20 \%$ of users and are mild to moderate in severity. Less common secondary effects include muscle cramps, decreased heart rate (bradycardia), decreased appetite and weight, and increased gastric acid production [29].

Glutamate is a major excitatory NT of CNS, although excessive amounts in the brain can lead to cell death through a process called excitotoxicity which consists of the overstimulation of glutamate receptors. It acts on the glutamatergic system by blocking NMDA receptors and inhibiting their overstimulation by glutamate. Memantine has been shown to be moderately efficacious in the treatment of moderate to severe Alzheimer's disease. Its effects in the initial stages of $\mathrm{AD}$ are unknown (Figure 5).

$A \beta$ facilitates endocytosis of glutamatergic receptors: NMDA (N-Methyl-D-Aspartate) and AMPA (a-amino-3-hydroxy-5methyl-4-isoxazole propionic acid). $A \beta$ also binds to the receptors of p75 neurotrophin (p75NTr) and Brain-Derived Neurotrophic Factor (BDNFr), also known as the tyrosine kinase $\mathrm{B}$ receptor (trkBr), exacerbating a situation in which levels of BDNF and Nerve Growth Factor (NGF) are already suppressed. $A \beta$ impairs nicotinic Acetylcholine (ACh) receptor (nAChr) signaling and ACh release from the presynaptic terminal. Numbers of hippocampal synapses decrease in mild cognitive impairment in which remaining synaptic profiles show compensatory increases in size. APP denotes amyloid precursor protein, pCaMKII phosphorylated calcium-calmodulintrkAr dependent protein kinase2, pCREB phosphorylated cyclic AMP response-element-binding protein, tyrosine kinase A receptor (trkAr) and VGCC voltage-gated calcium channel.

Normally high levels of neurotrophin receptors in cholinergic neurons in the basal forebrain are severely reduced in late-stage of AD. Ernfors and Bramham [30] suggested that BDNF replacement is another option for the treatment of $\mathrm{AD}$. In these disorders, exposure to $A \beta$ inhibits key mitochondrial enzymes in the brain and in isolated mitochondria and cytochrome c oxidase is specifically attacked. The increase in mitochondrial superoxide radical formation and conversion into hydrogen peroxide cause oxidative stress, release of cytochrome c, and apoptosis. Dysfunctional mitochondria release oxidizing free radicals, and in $\mathrm{AD}$ and the normal aging brain, they cause considerable oxidative stress [18].

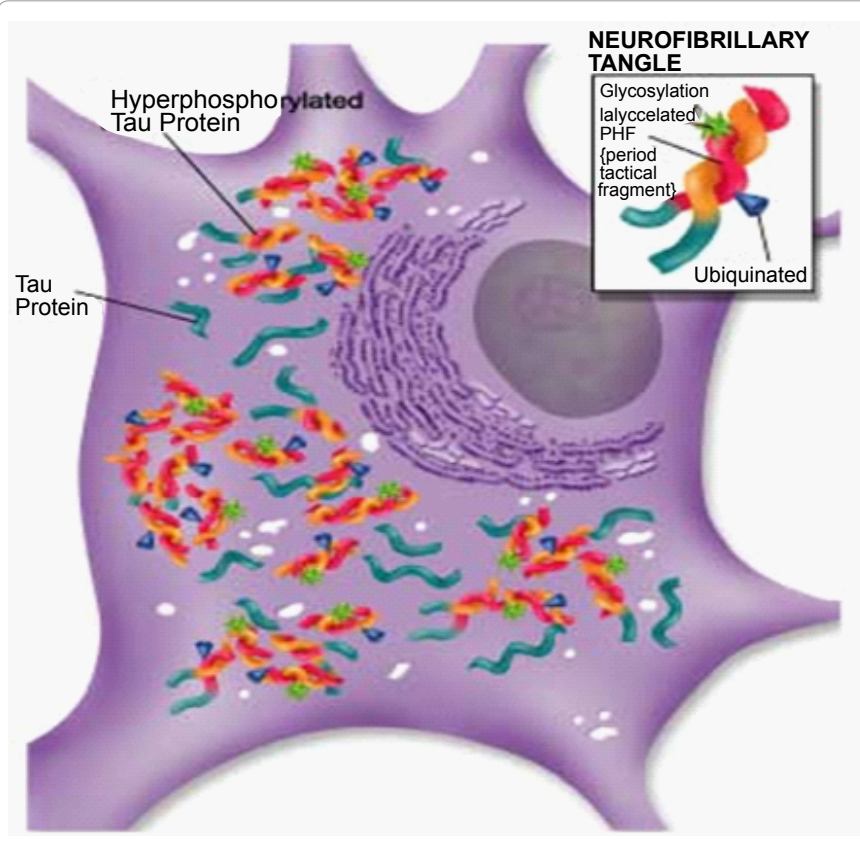

Microscopic image of a NFL, was forming by hyperphosphorylated tau protein The NFLs are abnormally composed or small protein of interconnected fibrils within neurons in AD. These balls are formed by multiple phosphorylation of microtubule-associated protein intracellular called tau. That hyperphosphorylation causes pathological features added soluble.

Figure 4: Tau structure and function. 
Cytokines also play critical roles in the development and progression of $\mathrm{AD}$. Cells associated with extracellular plaques within the brains of $\mathrm{AD}$ patients can produce a variety of cytokines and other related proteins that can ultimately influence plaque and tangle formation. Additionally, $A ß$ itself can stimulate microglia, astrocytes and oligodendrocytes to secrete proinflammatory cytokines, chemokines, and Reactive Oxygen Species (ROS) which can lead to neuronal damage. Several cytokines have been associated with $\mathrm{AD}$ development and progression, such as IL-1, IL-6, TGF- $\beta$ and TNF- $\alpha$. For example, a differential expression profile of various TGF- $B$ isotypes can be observed within $\mathrm{AD}$ plaques, neuronal tangles and the cells associated with senile plaques, thus suggesting a role for these cytokines in promoting lesion development [18].

\section{Familial Early-Onset of Alzheimer's Disease}

Further evidence comes from the finding that transgenic mice that express a mutant form of the human APP gene develop fibrillar amyloid plaques and Alzheimer's-like brain pathology with spatial learning deficits [31,32]. One approach involves examining individuals from Families with early-onset AD (FAD). FAD is characterized by autosomal dominant inheritance and a specific age at onset for a given pedigree [33]. Studies have been alerted on normal elderly at genetic risk for $\mathrm{AD}$, as determined by their being carriers of known susceptibility genes for late-life $\mathrm{AD}$ such as the ApoE, E4 allele [34]. Late-onset/ sporadic $\mathrm{AD}$ has virtually identical pathology to early-onset/familial $\mathrm{AD}$ (FAD), thus suggesting common pathogenic pathways for both forms of AD. To date, genetic studies have revealed four genes that may be linked to autosomal dominant or familial early-onset AD (FAD). These four genes include: Amyloid Precursor Protein (APP), Presenilin
1 (PS1), Presenilin 2 (PS2), and Apolipoprotein E (ApoE) [18]. All mutations associated with APP and PS proteins can lead to an increase in the production of $A ß$ peptides, specifically the more amyloidogenic form, Aß42. In addition to genetic influences on amyloid plaque and intracellular tangle formation, environmental factors (e.g., cytokines, neurotoxins, etc.) may also play important roles in the development and progression of $\mathrm{AD}$.

\section{Cause and Progression of Alzheimer's disease}

The cause and progression of AD disease are not well understood. Research indicates that the disease is associated with plaques and tangles in the brain [7]. Current treatments only help with the symptoms of the disease. There are no available treatments that stop or reverse the progression of the disease. More research needed on ways to prevent Alzheimer's, panel finds, more than 1000 clinical trials have been or are being conducted to find ways to treat the disease, but it is unknown if any of the tested treatments will work. Mental stimulation, exercise, and a balanced diet have been suggested as ways to delay cognitive symptoms (though not brain pathology) in healthy older individuals, but there is no conclusive evidence supporting an effect [18].

\section{Conclusions}

Both neuropathology and neuroimaging studies converge on observations that the pathological events implicated in AD occur early during the course of the disease and precede clinical symptoms. Moreover, the progression of $\mathrm{AD}$ pathology in the brain can be tracked using neuroimaging techniques. Over the past years, numerous studies have shown that brain atrophy and hypometabolism within

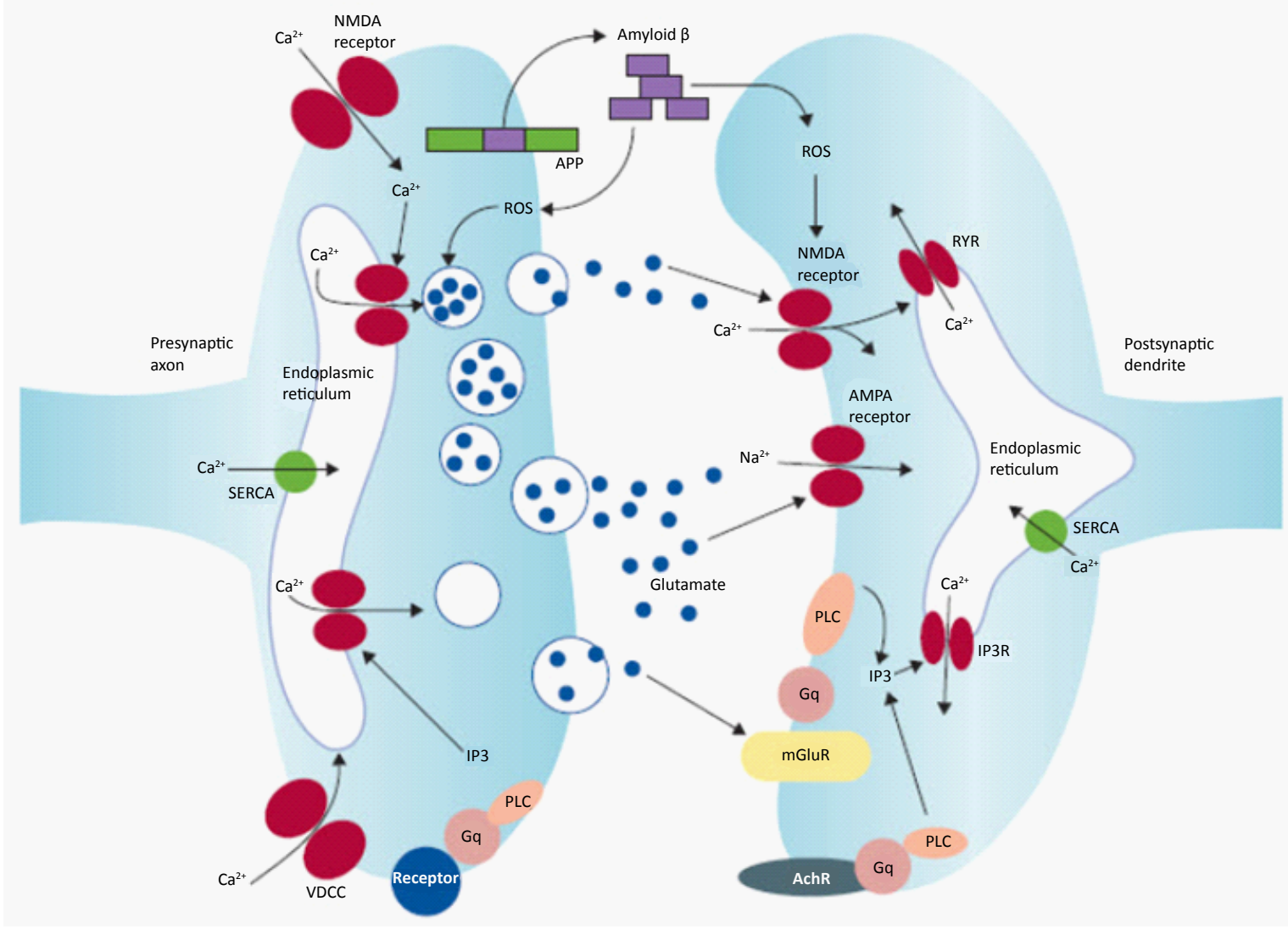

Figure 5: Synaptic Dysfunction in Alzheimer's Disease. 


\section{NEURODEGENERATIVE DISEASES}

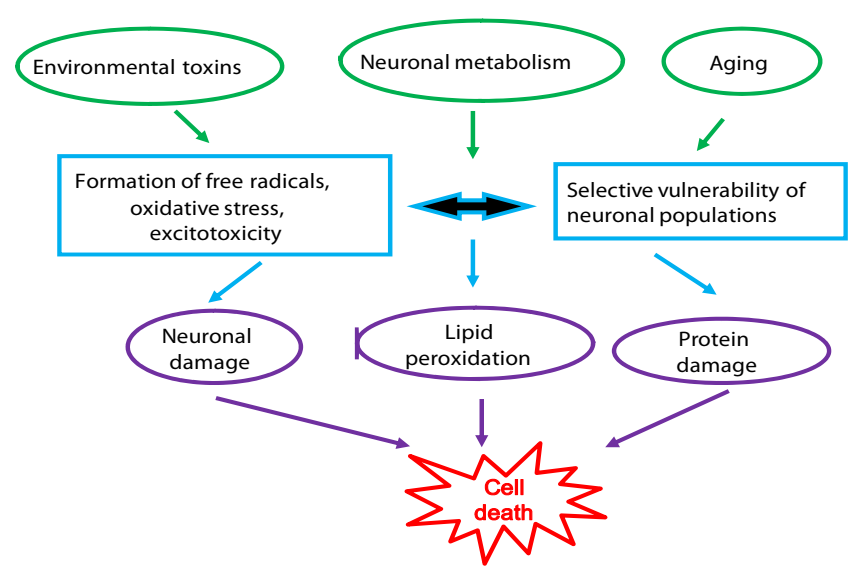

Figure 6: Neurodegenerative diseases leading to cell death.

key brain regions accurately distinguish $\mathrm{AD}$ from normal aging and other dementias. These pioneering data promoted the examination of neuroimaging in the preclinical detection of $\mathrm{AD}$.

An effective treatment for sporadic Alzheimer's disease rests on the translation of the disease pathways we have discussed, as well as additional molecular mechanisms or new risk genes defined by gene-expression profiling and whole-genome association studies into specific pharmacologic targets (Figure 6).

\section{References}

1. Cohen G. Age differences in memory for texts: production deficiency or processing limitations? L. Light, D. Burke (Eds.), Language, Memory and Aging, Cambridge University Press, New York (1990), pp. 171-190

2. Convit A, de Leon MJ, Tarshish C, De Santi S, Kluger A, et al. (1995) Hippocampal volume losses in minimally impaired elderly. Lancet 345: 266.

3. Convit A, de Asis J, de Leon MJ, Tarshish CY, De Santi S, et al. (2000) Atrophy of the medial occipitotemporal, inferior, and middle temporal gyri in non-demented elderly predict decline to Alzheimer's disease. Neurobiol Aging 21: $19-26$

4. Brookmeyer R, Gray S, Kawas C (1998) Projections of Alzheimer's disease in the United States and the public health impact of delaying disease onset. Am J Public Health 88: 1337-1342.

5. Waldemar G (2007) Recommendations for the diagnosis and management of Alzheimer's disease and other disorders associated with dementia: EFNS guideline. Eur J Neurol 14:1-26.

6. Tabert MH, Liu X, Doty RL, Serby M, Zamora D, et al. (2005) A 10-item smell identification scale related to risk for Alzheimer's disease. Ann Neurol 58: 155160.

7. Tiraboschi P, Hansen LA, Thal LJ, Corey-Bloom J (2004) The importance of neuritic plaques and tangles to the development and evolution of AD. Neurology 62: 1984-1989.

8. Braak H, Braak E (1991) Demonstration of amyloid deposits and neurofibrillary changes in whole brain sections. Brain Pathol 1: 213-216.

9. Braak H, Braak E (1996) Development of Alzheimer-related neurofibrillary changes in the neocortex inversely recapitulates cortical myelogenesis. Acta Neuropathol 92: 197-201.

10. Morris JC, Storandt M, McKeel DW Jr, Rubin EH, Price JL, et al. (1996) Cerebral amyloid deposition and diffuse plaques in "normal" aging: Evidence for presymptomatic and very mild Alzheimer's disease. Neurology 46: 707-719.

11. Delacourte A, David JP, Sergeant N, Buée L, Wattez A, et al. (1999) The biochemical pathway of neurofibrillary degeneration in aging and Alzheimer's disease. Neurology 52: 1158-1165.
12. Scarpini E, Scheltens P, Feldman H (2003) Treatment of Alzheimer's disease: current status and new perspectives. Lancet Neurol 2:539-547.

13. Ball MJ, Fisman M, Hachinski V, Blume W, Fox A, et al. (1985) A new definition of Alzheimer's disease: a hippocampal dementia. Lancet 1: 14-16.

14. Price JL, Morris JC (1999) Tangles and plaques in nondemented aging and "preclinical" Alzheimer's disease. Ann Neurol 45: 358-368.

15. Morrison JH, Hof PR (1997) Life and death of neurons in the aging brain Science 278: 412-419

16. de Leon MJ, Convit A, Wolf OT, Tarshish CY, DeSanti S, et al. (2001) Prediction of cognitive decline in normal elderly subjects with 2-[(18)F]fluoro-2-deoxy-Dglucose/poitron-emission tomography (FDG/PET). Proc Natl Acad Sci U S A 98: 10966-10971.

17. Chételat G, Desgranges $B$, de la Sayette V, Viader F, Eustache F, et al. (2003) Mild cognitive impairment: Can FDG-PET predict who is to rapidly convert to Alzheimer's disease? Neurology 60: 1374-1377.

18. Querfurth HW and La Ferla FM (2010). Alzheimer's disease. N Engl J Med 362:329-344.

19. Lee VM, Goedert M, Trojanowski JQ (2001) Neurodegenerative tauopathies Annu Rev Neurosci 24: 1121-1159.

20. Schmidt ML, Zhukareva V, Newell KL, Lee VM, Trojanowski JQ (2001) Tau isoform profile and phosphorylation state in dementia pugilistica recapitulate Alzheimer's disease. Acta Neuropathol 101: 518-524.

21. Bobinski M, de Leon MJ, Convit A, De Santi S, Wegiel J, et al. (1999) MRI of entorhinal cortex in mild Alzheimer's disease. Lancet 353: 38-40.

22. Bobinski M, de Leon MJ, Wegiel J, Desanti S, Convit A, et al. (2000) The histological validation of post mortem magnetic resonance imaging-determined hippocampal volume in Alzheimer's disease. Neuroscience 95: 721-725.

23. Turner PR, O'Connor K, Tate WP, Abraham WC (2003) Roles of amyloid precursor protein and its fragments in regulating neural activity, plasticity and memory. Prog Neurobiol 70: 1-32

24. Priller C, Bauer T, Mitteregger G, Krebs B, Kretzschmar HA, et al. (2006) Synapse formation and function is modulated by the amyloid precursor protein J Neurosci 26: 7212-7221.

25. Polvikoski T, Sulkava R, Haltia M, Kainulainen K, Vuorio A, et al. (1995) Apolipoprotein $\mathrm{E}$, dementia, and cortical deposition of beta-amyloid protein. $\mathrm{N}$ Engl J Med 333: 1242-1247.

26. Oddo S, Caccamo A, Shepherd JD, et al. (2003)Triple-transgenic model of Alzheimer's of daily living, behaviour, and global function in patients with mildto-moderate.

27. Raschetti R, Albanese E, Vanacore N, Maggini M (2007) Cholinesterase inhibitors in mild cognitive impairment: a systematic review of randomised trials. PLoS Med 4: e338.

28. Li G, Faibushevich A, Turunen BJ, Yoon SO, Georg G, et al. (2003) Stabilization of the cyclin-dependent kinase 5 activator, p35, by paclitaxel decreases betaamyloid toxicity in cortical neurons. J Neurochem 84: 347-362.

29. Mosch B, Morawski M, Mittag A, Lenz D, Tarnok A, et al. (2007) Aneuploidy and DNA replication in the normal human brain and Alzheimer's disease. $J$ Neurosci 27: 6859-6867.

30. Ernfors P, Bramham CR (2003) The coupling of a trkB tyrosine residue to LTP. Trends Neurosci 26: 171-173.

31. Hsiao K, Chapman P, Nilsen S, Eckman C, Harigaya Y, et al. (1996) Correlative memory deficits, Abeta elevation, and amyloid plaques in transgenic mice. Science 274: 99-102.

32. Lalonde R, Dumont M, Staufenbiel M, Sturchler-Pierrat C, Strazielle C (2002) Spatial learning, exploration, anxiety, and motor coordination in female APP23 transgenic mice with the Swedish mutation. Brain Res 956: 36-44.

33. Tanzi RE, Bertram L (2001) New frontiers in Alzheimer's disease genetics Neuron 32: 181-184

34. Corder EH, Saunders AM, Strittmatter WJ, Schmechel DE, Gaskell PC, et al. (1993) Gene dose of apolipoprotein E type 4 allele and the risk of Alzheimer's disease in late onset families. Science 261: 921-923. 